\title{
Altered PTEN expression as a diagnostic marker for the earliest endometrial precancerous changes
}

\author{
Sharda B. ${ }^{1}$, Malik R. ${ }^{2}$, Jain $\mathbf{P .}^{3}$ \\ ${ }^{1}$ Dr. Bela Sharda PG Resident, ${ }^{2}$ Dr. Reeni Malik, Professor and Head of Department, ${ }^{3}$ Dr. Pramila Jain, Associate \\ Professor. All authors are affiliated with Department of Pathology, Gandhi Medical College, Bhopal, Madhya Pradesh, \\ India.
}

Address of Correspondence: Dr. Bela Sharda, 7/230 M.G. Road, Sonkatch, District Dewas, Madhya Pradesh, India. E-mail: dr.bela22@gmail.com

\begin{abstract}
Introduction: Study was planned to investigate PTEN gene expression in endometrial hyperplasia and carcinoma as analyzed by immunohistochemistry. Material and Method: This study was conducted on 80 endometrial samples in the Department of Pathology, Gandhi Medical College, Bhopal (January 2012 to August 2016) Results: 5 out of 5 cases of proliferative endometrium, 3 out of 5 cases of secretory endometrium, 37 [84\%] out of 44 cases of simple and complex endometrial hyperplasia without atypia, 8 out of 15 cases of simple and complex endometrial hyperplasia with atypia and 3 out of 11 cases of endometrial carcinoma showed positive PTEN expression. Conclusion: PTEN is a major gene involved in the pathogenesis of endometrial carcinoma. Our data suggest that decreased PTEN expression was a marker of earliest endometrial pre-cancer lesion and we propose that use of PTEN immunostaining in a clinical setting may be informative in identifying premalignant lesions that are likely to progress to carcinoma.
\end{abstract}

Keywords: PTEN, Endometrial hyperplasia, Endometrial carcinoma

\section{Introduction}

Endometrial carcinoma is one of the most common invasive neoplasm of the female genital tract [1]. A recent cancer registry [1994-2011] reported that malignancies of the corpus uteri comprised $3.02 \%$ of all neoplasm in adult females [2]. Endometrioid Endometrial Carcinoma accounts for three fourth of endometrial cancers and are thought to develop following a continuum of premalignant lesions ranging from endometrial hyperplasia without atypia, hyperplasia with atypia and well differentiated carcinoma [3,4]. Based on light microscopic appearance and clinical behaviour, endometrial cancers have been classified into two major categories. (type I and II) [46]. Accurate diagnosis of premalignant lesions in routine endometrial biopsies has a great clinical value in patient management. Recent molecular diagnostic methods have provided new ancillary tools for

Manuscript received: $04^{\text {th }}$ May 2017

Reviewed: $12^{\text {th }}$ May 2017

Author Corrected: $20^{\text {th }}$ May 2017

Accepted for Publication: $31^{\text {st }}$ May 2017 premalignant lesion diagnosis. EECA and atypical hyperplasias has a variety of genetic alternations, including microsatellite instability (MI) and mutations of PTEN, k-ras, and $\beta$-catenin genes [7,8]. Currently, PTEN is the most frequently altered gene in EECA which is located on chromosome 10 [7,8]. The PTEN gene has both lipid and protein phosphate activity and the combination of the losses of PTEN lipid and protein phosphate activity can cause an aberrant cell growth and an escape from apoptosis, as well as abnormal cell spreading and migration [8]. Up to $50 \%$ of all EECA and $83 \%$ of tumors with adjacent premalignant lesions show altered PTEN, characterized by loss of expression.[7-9] Mutations of PTEN are frequently detected in several cancers such as: endometrium, low grade endometrioid ovarian carcinoma (20\%), prostate, breast and glial tumors [10-20]. Among the different histological subtypes of EECA, endometrial subtypes have the highest frequency $(34-80 \%)$ of PTEN mutations [21]. PTEN - null glands (i.e., loss of PTEN expression) are shown in a diffuse pattern in EECA but also may be detected in morphologically normal 
endometrial tissue, which suggests that PTEN alternation occur in the earliest phase of endometrial carcinogenesis [7,19,22]. Immunohistochemical detection of PTEN in cycling endometrium reveals high levels of protein expression in all different cell types during the proliferative phase, with diminution or absence of PTEN protein expression in mid secretory glands $[3,19,23,24]$. The hypothesis that loss of PTEN expression could be assessed by immunohistochemical method has led to the suggestion that PTEN immunostaining may be a new and effective tool for screening of malignant and premalignant endometrial lesions [13,25]. In the present study we used immunohistochemical method to evaluate PTEN expression in three groups of specimens from normal, hyperplastic endometrium and EECA.

\section{Material and Method}

This study was conducted to evaluate the histopathological pattern and its immunohistochemical expression correlation with phosphotensin tumour suppressor gene staining pattern by PTEN marker in endometrial hyperplasia and carcinoma.

Study design- Retrospective and prospective study Setting- Histology section of Department of Pathology, Gandhi Medical College and Hamidiya Hospital, Bhopal, M.P

Inclusion criteria - All the endometrial biopsies and hysterectomy specimens diagnosed as endometrial hyperplasia and carcinomas were selected for study.

Exclusion criteria - Autolysed samples, biopsies with predominantly blood clots, very tiny bit of tissue inadequate for opinion, cervical tissue instead of endometrium. Specimen with any evidence of endometritis were excluded.

Participants - Cases diagnosed as endometrial hyperplasia and endometrial carcinoma were included in the study.
Variables - Intensity of color reaction and slide area staining.

Data source - All the endometrial biopsies and hysterectomy specimens diagnosed as endometrial hyperplasia and carcinomas in the Department of Pathology, Gandhi Medical College from January 2012 to August 2016 were selected for study.

Bias - Random selection.

Study size - Out of a total 265 endometrial tissue samples observed, 80 samples were selected randomly for immunohistochemistry, including 5 normal proliferative endometrium, 5 normal secretory endometrium, 40 simple hyperplasia without atypia, 8 simple hyperplasia with atypia,4 complex hyperplasia without atypia, 7 complex hyperplasia with atypia,11 endometrial carcinomas in the study. Samples were selected for immunohistochemistry analysis on the basis of clinical presentation and histological diagnosis according to WHO histological classification. The most representative paraffin block for each case was then selected for immunohistochemistry analysis

Statistical analysis - The chi square test was applied and $\mathrm{p}$-value $<0.05$ was considered significant at $95 \%$ confidence interval.

Evaluation of immunostaining- Immunoreactivity was regarded as positive when brown staining was localized in the nuclei or cytoplasm of normal endometrial glandular cell (an internal positive control) or tumour cell. According to Kapucuoglu et al and An et al the immunoreactivity was graded arbitrarily and semi quantitatively by considering the percentage and intensity of staining on the whole section $[26,27]$.

$$
\begin{aligned}
& \text { Intensity of staining } \\
& 0=\text { absent } \\
& +1=\text { light brown } \\
& +2=\text { dark brown }
\end{aligned}
$$

\section{Percentage of staining}

Negative $-<10 \%$ of the slide's area stained.

$+1-10-50 \%$ of the slide's area stained.

$+2->50 \%$ of the slide's area stained.

\section{Results}

In the present study, out of 80 cases, 56 cases [70\%] showed PTEN positive expression and 24 cases were negative for PTEN expression. Proliferative endometrium showed $100 \%$ positivity followed by simple hyperplasia without atypia [87.5\%].Least positivity was seen with endometrial carcinoma i.e. only 3 cases. [Table 1]

Out of all hyperplasias, simple hyperplasia without atypia predominates [67.79\%] followed by simple hyperplasia with atypia [13.55\%]. [Table 2] 
Table-1: Distribution of cases for Immunohistochemical evaluation [N=80]

\begin{tabular}{|c|c|c|}
\hline Histopathological diagnosis & IHC no. of cases & Positive IHC \\
\hline Proliferative endometrium & 5 & 5 \\
\hline Secretory endometrium & 5 & 3 \\
\hline Simple hyperplasia without atypia & 40 & 4 \\
\hline Simple hyperplasia with atypia & 8 & 2 \\
\hline Complex hyperplasia without atypia & 4 & 4 \\
\hline Complex hyperplasia with atypia & 7 & 3 \\
\hline Endometrial carcinoma & 11 & $\mathbf{5 6}[\mathbf{7 0} \%]$ \\
\hline
\end{tabular}

Table-2: Histopathological types of Endometrial Hyperplasia [N=80]

\begin{tabular}{|c|c|c|}
\hline Variants of endometrial hyperplasia & Number of cases & Percentage [\%] \\
\hline Simple hyperplasia without atypia & 40 & $67.79 \%$ \\
\hline Simple hyperplasia with atypia & 8 & $13.55 \%$ \\
\hline Complex hyperplasia without atypia & 4 & $6.77 \%$ \\
\hline Complex hyperplasia with atypia & 7 & $11.86 \%$ \\
\hline Total & $\mathbf{5 9}$ & $\mathbf{1 0 0} \%$ \\
\hline
\end{tabular}

\section{Intensity of staining - [Table 3]}

In normal endometrium - Proliferative endometrium - Out of 5 cases, 3 cases showed score +1 [light brown] and 2 cases showed +2 score [dark brown]. All cases showed positive staining with PTEN in proliferative endometrium. Secretory endometrium - Out of 5 cases, 2 cases showed score 0 [absent staning] and 3 cases showed +1 score [light brown]. None of the secretory endometrium showed +2 or dark brown staining. 3 cases in secretory endometrium showed positive PTEN expression.

In hyperplasia- In simple hyperplasia without atypia - Out of 40 cases, 5 cases showed score 0 [absent staining], 35 cases [87.5\%] showed score +1 [light brown] and 15 cases showed +2 score [dark brown] with PTEN staining. In simple hyperplasia with atypia - out of 8 cases, 4 cases showed score 0 [absent staining], 3 cases showed score +1 [light brown] and only 1 case showed +2 score [dark brown] with PTEN staining. In complex hyperplasia without atypia - Out of 4 cases, 2 cases showed score 0 [absent staining] and 2 cases showed score +1 [light brown]. None of the cases showed +2 score [dark brown] with PTEN staining. In complex hyperplasia with atypia -Out of 7 cases, 3 cases showed score 0 [absent staining], 4 cases showed score +1 [light brown].

In endometrial carcinoma- Out of 11 cases, 8 cases showed score 0 [absent staining], 2 cases showed score +1 [light brown] and only 1 case showed +2 score [ dark brown] with PTEN staining. Thus, PTEN expression was significantly higher with strong color intensity in proliferative endometrium than in atypical hyperplasia and endometrial carcinoma.

\section{Percentage of staining - [Table 4]}

In normal endometrium-Proliferative endometrium - Out of 5 cases, 3 cases showed score +1 and 2 cases showed +2 score. $100 \%$ cases shows positive staining with PTEN in proliferative endometrium. Secretory endometrium - Out of 5 cases, 3 cases showed score 0 and 2 cases showed +1 score. None of the secretory endometrium showed +2 score.

\section{In hyperplasia -}

In simple hyperplasia without atypia - Out of 40 cases, 5 cases showed score 0,25 cases showed score +1 and 10 cases showed +2 score. In simple hyperplasia with atypia - Out of 8 cases, 4 cases showed score 0,3 cases showed score +1 and only 1 case showed +2 score. 
In complex hyperplasia without atypia - Out of 4cases, 2 cases showed score 0 and 2 cases showed score +1 . None of the cases showed +2 score .In complex hyperplasia with atypia - Out of 7 cases, 3 cases showed score 0,3 cases showed score +1 and only 1 case showed +2 score.

In endometrial carcinoma- Out of 11 cases, 8 cases showed score 0,3 cases showed score +1 and none of the cases showed +2 score. We found that most of the cases showed score +2 [ $>50 \%$ of slide's area stained] in proliferative endometrium as compared to atypical hyperplasia and carcinoma.

Thus, PTEN expression was significantly higher in proliferative endometrium than in atypical hyperplasia and endometrial carcinoma. This shows that PTEN inactivation initiate in precancers from a normal background state, and additional PTEN damage accumulates in the transition from premalignant to malignant disease.

Table-3: PTEN expression based on the Intensity of color reaction

\begin{tabular}{|c|c|c|c|c|}
\hline Endometrial lesions & $\mathbf{0}$ & $\mathbf{+ 1}$ & $\mathbf{+ 2}$ & Total no. of cases \\
\hline Proliferative endometrium & 0 & 3 & 2 & 5 \\
\hline Secretory endometrium & 2 & 3 & 0 & 5 \\
\hline Simple hyperplasia without atypia & 5 & 20 & 15 & 40 \\
\hline Simple hyperplasia with atypia & 4 & 3 & 1 & 8 \\
\hline Complex hyperplasia without atypia & 2 & 2 & 0 & 4 \\
\hline Complex hyperplasia with atypia & 3 & 4 & 0 & 11 \\
\hline Endometrial carcinoma & 8 & 2 & 1 & 7 \\
\hline
\end{tabular}

Table-4: PTEN expression based on the Slide's area staining

\begin{tabular}{|c|c|c|c|}
\hline Type of endometrium & $\begin{array}{c}<\mathbf{1 0} \% \\
{[\text { score 0] }}\end{array}$ & $\begin{array}{c}\mathbf{1 0 - 5 0 \%} \\
\text { [score +1] }\end{array}$ & $\begin{array}{c}>\mathbf{5 0} \% \\
\text { [score +2] }\end{array}$ \\
\hline Proliferative endometrium & 0 & 3 & 2 \\
\hline Secretory endometrium & 3 & 2 & 0 \\
\hline Simple hyperplasia without atypia & 5 & 25 & 10 \\
\hline Simple hyperplasia with atypia & 4 & 3 & 0 \\
\hline Complex hyperplasia without atypia & 2 & 2 & 1 \\
\hline Complex hyperplasia with atypia & 3 & 3 & 0 \\
\hline Endometrial carcinoma & 8 & 3 & \\
\hline
\end{tabular}

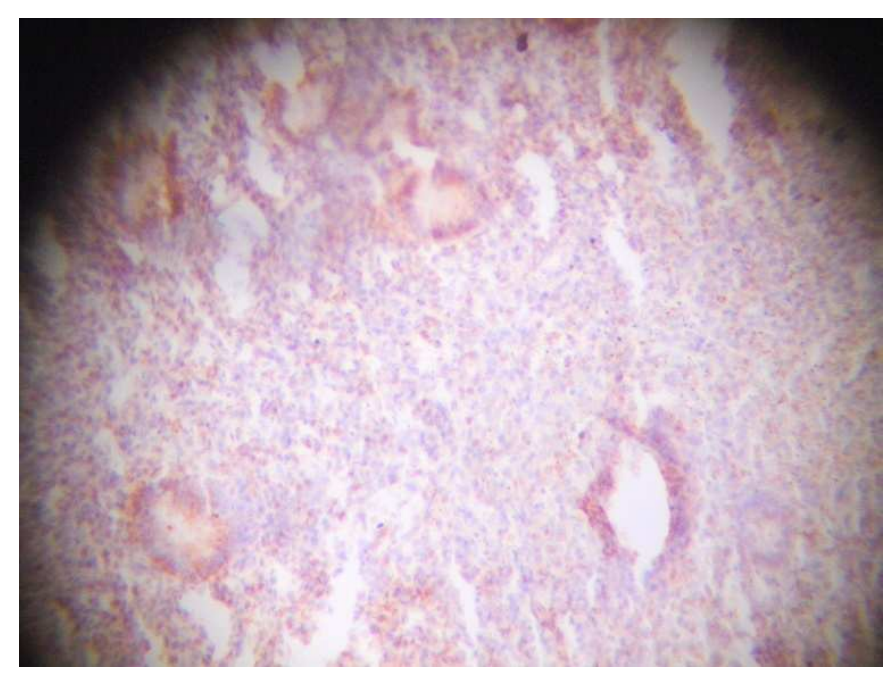

Figure-1: IHC stained slide - Low power view - Proliferative Endometrium 


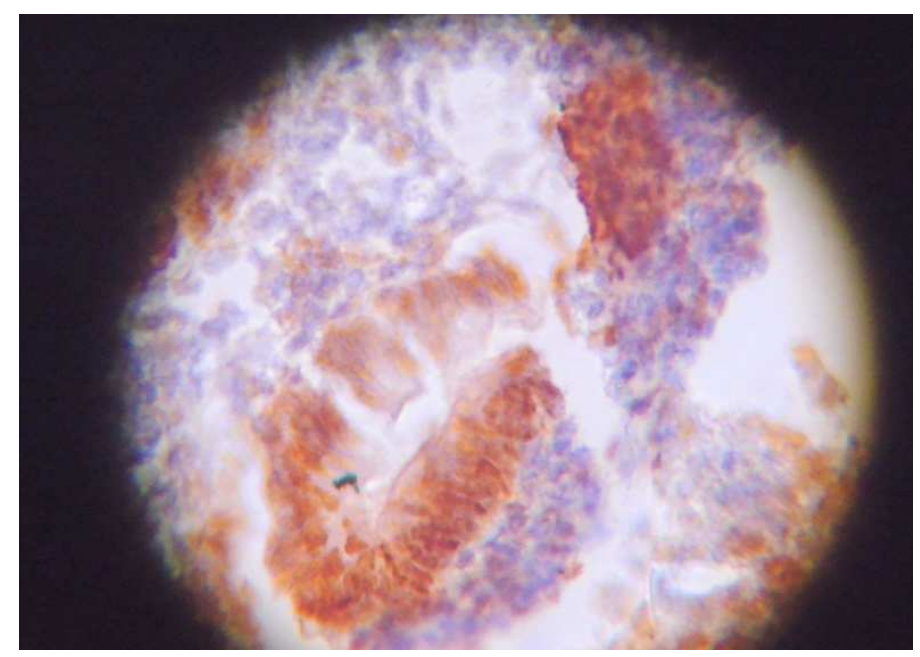

Figure-2: IHC stained slide - High power view - Proliferative Endometrium

\section{Discussion}

All 5 cases of proliferative endometrium shows PTEN positivity while 3 out of 5 cases of secretory endometrium shows PTEN positivity. The intensity varied from moderate to strong in proliferative phase while absent to mild in secretory phase. The difference between two groups is statistically insignificant.

We also found a significantly higher PTEN expression in EH [84\%] than in EC [27.27\%] [p <0.05] while no statistically significant difference was seen between $\mathrm{AH}$ and EC [ $p>0.05]$.

In our study, $72.7 \%$ of EC and $46.6 \%$ of AH shows loss of PTEN expression. Out of 11 cases of EC, 8 cases showed totally absent PTEN expression, 2 showed weak to moderate and 1 case showed strong staining with PTEN.

Mutter et al [2000] found that PTEN expression in 61\% (20 of 33) of cases was completely absent and 97\% (32 of 33) of cases revealed at least some diminution in expression. He also found that somatic PTEN mutations were detected in $83 \%$ of endometrial carcinoma and in $55 \%$ in premalignant lesions, and the difference was statistically significant $(\mathrm{P}=0.025)$. [28]

Erkanli S et al [2006] observed that PTEN expression was decreased in hyperplasia [14 cases out of 38, 36.8\%] and carcinoma [12 cases out of 29, 41.4\%] cases with respect to proliferative endometrium [100\%][29].

Patau Tantbirojn MD et al [2008] observed moderate to strong PTEN immunoreactivity in proliferative endometrium and absent or mild PTEN expression was in the secretory endometrium [30].

Sohelia Sarmadi et al [2009] detected loss of PTEN expression in 52\% of EC and 25\% of AH They also observed that PTEN positivity was present in all proliferative endometrium [100\%]with no differences between early and late proliferative phases, and the highest PTEN immunoreactivity as well as homogeneity were detected in normal proliferative endometrium [31].

Nehad M.R. Abd El-Maqsoud et al [2009] observed that loss of PTEN immunoreactivity was 48.6\% in EC.They also noticed a higher, although not significant PTEN staining score in the proliferative endometrium than in the secretory endometrium. No significant differences were detected between PTEN expression in proliferative phase and secretory phase [32]. 
Samah et al [2011] PTEN expression was observed in a total of 33 cases out of the total 50 cases with proliferative endometrial lesions $(66 \%)$ [34].

Heejeong Lee et al [2012] Altered PTEN expression was noted in 2 of 10 normal endometrium (20\%) and 80\% cases shows positive PTEN expression [35].

Summaya Shawana et al [2016] All 4 normal proliferative endometrial samples showed moderate to strong PTEN staining.[100\%] [36]. Orbo et al [2003] reported loss of PTEN protein expression in 55\% of specimens in patients with subsequent EC [37].

Cirpan et al [2006] examined PTEN protein immunoreactivity in cases with endometrial adenocarcinoma and found that none of the 10 cases of endometrial carcinomas showed absent PTEN expression. In addition, complete loss of PTEN immunoreactivity was found in only 1 out of 24 cases with EIN [38].

Kapucuoglu et al [2007] found complete loss of PTEN in 20\% of atypical complex hyperplasia and PTEN immunoreactivity was present in all hyperplasia without atypia and in 48\% of EC.Kapucuoglu et al [2007] and Nehad M.R. Abd El-Maqsoud et al [2009] suggested that AH is the precursor of EC .Common histopathologic criteria between $\mathrm{EC}$ and $\mathrm{AH}$ are present, therefore there was no statistically significant differences in PTEN expression levels between AH and EC. Previous molecular studies have found that $34-83 \%$ of all tumors and 55\% of endometrial intraepithelial hyperplasias, which are regarded as precancer lesions, harbor point mutations or deletions within the PTEN gene, indicating that PTEN inactivation is an early event in endometrial carcinogenesis [39].

Allison et al [2008] showed that biomarkers alone or in combination had most consistency to make a clear distinction between normal,endometrial hyperplasia and EC. They found reduced PTEN expression in 52\% of EC [40].

Table-5: Comparison of PTEN positivity percentage with previous studies

\begin{tabular}{|c|c|c|c|c|c|}
\hline Studies & No.of cases & $\begin{array}{c}\text { Normal } \\
\text { endometrium }\end{array}$ & $\begin{array}{c}\text { Simple } \\
\text { hyperplasia }\end{array}$ & $\begin{array}{c}\text { Atypical } \\
\text { hyperplasia }\end{array}$ & Carcinomas \\
\hline George L[2000] $^{28}$ & 89 & $95 \%$ & - & $25 \%$ & $39 \%$ \\
\hline S.Erkanli[2006] $^{29}$ & 77 & $100 \%$ & $63.2 \%$ & & $58.6 \%$ \\
\hline Patau [2008] & 70 & & $76 \%$ & $40 \%$ & $40 \%$ \\
\hline Sohelia [2009] & 87 & $100 \%$ & $100 \%$ & $75 \%$ & $48 \%$ \\
\hline Nehad [2009] ${ }^{32}$ & 67 & $100 \%$ & $100 \%$ & $75 \%$ & $51.4 \%$ \\
\hline Afaf[2011] & 53 & $25 \%$ & $58 \%$ & $50 \%$ & $28.57 \%$ \\
\hline Samah [2011] $]^{33}$ & 50 & - & $100 \%$ & - & $40 \%$ \\
\hline Heejeong [2012] & 75 & $80 \%$ & $76 \%$ & $29 \%$ & $32 \%$ \\
\hline Summayya[2016] $]^{36}$ & 53 & $100 \%$ & $95 \%$ & $34.4 \%$ & $52.2 \%$ \\
\hline Present study & 80 & $80 \%$ & $84 \%$ & $53.33 \%$ & $27.27 \%$ \\
\hline
\end{tabular}

Previous studies showed that hyperplasia with atypia may develop into endometrial carcinoma and loss of PTEN function by mutation or other mechanisms was an early event in endometrial tumorigenesis that may occur in response to known endocrine risk factors and offers an informative immunohistochemical biomarker for premalignant disease, we used immunohistochemical method to evaluate PTEN expression in three groups of specimens from normal endometrium, hyperplastic endometrium and endometrial carcinomas [41]. PTEN is a tumor suppressor gene located at chromosome 10q23 and it encodes a 55-KD protein with tyrosine kinase function. It acts at the G1/S checkpoint of the cell cycle and enables apoptosis through an AKT dependent mechanism. PTEN acts in opposition to PI3KCA to control levels of phosphorylated AKT and its mutation results in increased PI3KCA activity leading to increased AKT phosphorylation. PTEN immunoreactivity was detected in the nucleus with stromal reaction.

In present study,we found that high PTEN score was present in the proliferative phase, while low score in glandular epithelial cells seen in the secretory phase. There was low PTEN scores in most of the endometrial carcinoma and 
atypical hyperplasia as compared to simple hyperplasia. This showed that PTEN inactivation initiate in precancers from a normal background state, and additional PTEN damage accumulates in the transition from premalignant to malignant disease. This may explain the gradual decline in PTEN expression from benign to atypical hyperplasia and more severe decline in malignant cases.

Most of the cases of proliferative endometrium and simple hyperplasia showed moderate to strong intensity while atypical hyperplasia and endometrial carcinoma showed absent to weak staining with PTEN. Decreased PTEN expression tends to be associated with malignant endometrium with significant statistical difference of PTEN immunoreactivity between groups of normal endometrium, hyperplastic changes and carcinoma. From the present study, it can be concluded that loss of PTEN expression was a potential marker for predicting the subsequent risk of endometrial carcinoma. Thus, PTEN may be a useful immunohistochemical biomarker of mutations of the PTEN suppressor gene.

\section{Conclusion}

We believe that women with atypical endometrial hyperplasia and early onset endometrial carcinoma should be evaluated for PTEN expression in their endometrial biopsies. Such an analysis could have therapeutic implications, given that inhibitors of the PTEN mutation pathway are being studied in patients with advanced endometrial cancer which will be beneficial to the patients.

\section{Abbreviations}

AH - Atypical Hyperplasia, EC - Endometrial carcinoma, EECA - Endometriod endometrial carcinoma, IHC- Immumohistochemistry, MI Microsatellite instability, PTEN -Phosphotensin tumor suppressor, WHO - World health organisation

\section{Funding: Nil, Conflict of interest: None Permission of IRB: Yes}

\section{References}

1. Jemal A, Bray F, Center MM, Ferlay J, Ward E, Forman D. Global cancer statistics. CA Cancer J Clin. 2011 Mar-Apr;61(2):69-90. doi: 10.3322/caac.20107. Epub 2011 Feb 4.

2. Badar F, Mahmood S. Hospital-based cancer profile at the Shaukat Khanum Memorial Cancer Hospital and Research Centre, Lahore, pakistan. J Coll Physicians Surg Pak. 2015 Apr;25(4):259-63. doi: 04.2015/JCPSP.259263.

3. Mutter GL. Histopathology of genetically defined endometrial precancers. Int J Gynecol Pathol. 2000 Oct;19(4):301-9.
4. Boruban MC, Altundag K, Kilic GS, Blankstein J: From endometrial hyperplasia to endometrial cancer: insight into the biology and possible medical preventive measures. Eur J Cancer Prev 2008 Apr;17(2):133-8.

5. Mutter GL. Diagnosis of premalignant endometrial disease. J Clin Pathol. 2002 May;55(5):326-31.

6. Hecht JL, Mutter GL. Molecular and pathologic aspects of endometrial carcinogenesis. J Clin Oncol. 2006 Oct 10;24(29):4783-91.

7. Kapucuoglu N, Aktepe F, Kaya H, Bircan S, Karahan N, Ciriş M. Immunohistochemical expression of PTEN in normal, hyperplastic and malignant endometrium and its correlation with hormone receptors, bcl-2, bax, and apoptotic index. Pathol Res Pract. 2007;203(3):153-62. Epub 2007 Feb 20.

8. Liu FS. Molecular carcinogenesis of endometrial cancer. Taiwan J Obstet Gynecol. 2007 Mar;46(1):2632.

9. Mutter GL, Ince TA, Baak JP, Kust GA, Zhou XP, Eng C: Molecular identification of latent precancers in histologically normal endometrium. Cancer Res 2001, 61:4311-4.

10. An HJ, Lee YH, Cho NH, Shim JY, Kim JY, Lee C, et al: Alteration of PTEN expression in endometrial carcinoma is associated with down-regulation of cyclin dependent kinase inhibitor,p27. Histopathology 2002, Nov 41(5):437-45. 
11. Bussaglia E, del Rio E, Matias-Guiu X, Prat J: PTEN mutations in endometrial carcinomas: a molecular and clinicopathologic analysis of 38 cases. Human Pathol 2000, 31:312-7.

12. Kanamori Y, Kigawa J, Itamochi H, Shimada M, Takahashi M, KamazawaS, et al.: Correlation between loss of PTEN expression and Akt phosphorylation in endometrial carcinoma. Clin Cancer Res 2001, 7:892-5.

13. Mutter GL, Lin MC, Fitzgerald JT, Kum JB, Baak JP, Lees JA, et al.:Altered PTEN expression as a diagnostic marker for the earliest endometrial precancers. J Natl Cancer Inst 2000,Jun 7; 92:924-30.

14. Orbo A, Nilsen MN, Arnes MS, Pettersen I, Larsen K. Loss of expression of MLH1, MSH2, MSH6, and PTEN related to endometrial cancer in 68 patients with endometrial hyperplasia. Int $\mathrm{J}$ Gynecol Pathol. 2003 Apr;22(2):141-8.

15. Salvesen HB, Stefansson I, Kalvenes MB, Das $\mathrm{S}$, Akslen LA. Loss of PTEN expression is associated with metastatic disease in patients with endometrial carcinoma. Cancer. 2002 Apr 15;94(8):2185-91.

16. Geyer JT, López-García MA, Sánchez-Estevez C, Sarrió D, Moreno-Bueno G, Franceschetti I, Palacios J, Oliva E. Pathogenetic pathways in ovarian endometrioid adenocarcinoma: a molecular study of 29 cases. Am J Surg Pathol. 2009 Aug;33(8):1157-63. doi: 10.1097/PAS.0b013e3181a902e1.

18. Visakorpi T: The molecular genetics of prostate cancer. Uorology 2003 Nov, 62:3-9.

19. Bose S, Crane A, Hibshoosh H, Mansukhani M, Sandweis L, Parsons R: Reduced expression of PTEN correlates with breast cancer progression. Hum Pathol 2002, 33:405-9.

20. Kimura F, Watanabe J, Hata H, Fujisawa T, Kamata Y, Nishimura Y, et al.: PTEN immunohistochemical expression is suppressed in G1 endometrioid adenocarcinoma of the uterine corpus. J Cancer Res Clin Oncol 2004, 130:161-8.
21. Tashiro H, Blazes MS, Wu R, Cho KR, Bose S, Wang SI, et al.: Mutations in PTEN are frequent in endometrial carcinoma but rare in other common gynecological malignancies. Cancer Res 1997, Sep15,57:3935-40.

22. Tantbirojn $\mathrm{P}$, Triratanachat $\mathrm{S}$, Trivijitsilp $\mathrm{P}$, Niruthisard S: Detection of PTEN immunoreactivity in endometrial hyperplasia and adenocarcinoma. J Med Assoc Thai 2008 Aug, 91:1161-5.

23. Lacey JV Jr, Mutter GL, Ronnett BM, Ioffe OB, Duggan MA, Rush BB, Glass AG, Richesson DA, Chatterjee N, Langholz B, Sherman ME. PTEN expression in endometrial biopsies as a marker of progression to endometrial carcinoma. Cancer Res. 2008 Jul 15;68(14):6014-20. doi: 10.1158/00085472.CAN-08-1154.

24. Mutter GL: EIN Central.endometrium org October 29:1-25.

25. Mutter GL, Lin MC, Fitzgerald JT, Kum JB, Eng C. Changes in endometrial PTEN expression throughout the human menstrual cycle. J Clin Endocrinol Metab. 2000 Jun;85(6):2334-8.

26. Pallares J, Bussaglia E, Martínez-Guitarte JL, Dolcet $\mathrm{X}$, Llobet $\mathrm{D}$, Rue $\mathrm{M}$, et al.: Immunohistochemical analysis of PTEN in endometrial carcinoma: a tissue microarray study with a comparison of four commercial antibodies in correlation with molecular abnormalities. Mod Pathol 2005, 18:719-27.

27. Kapucuoglu N, Aktepe F, Kaya H, Bircan S, Karahan N, Ciris, M. Immunohistochemical expression of PTEN in normal, hyperplastic and malignant endometrium and its correlation with hormone receptors, bcl-2, bax, and apoptotic index. Pathol Res Pract. 2007, 203:153-62.

28. An HJ, Lee YH, Cho NH, Shim JY, Kim JY, Lee $\mathrm{C}$,et al. Alteration of PTEN expression in endometrialcancer is associated with down-regulation of cyclin dependent kinase inhibitor, p27. Histopathology.2002 Nov, 41: 437-45208.

29. Mutter GL, Lin MC. Fitzgerald JT, et al. Altered PTEN expression as a diagnostic marker for the earliest 
endometrial precancers. J Natl Cancer lnst 2000 Jun 7;92:924-30

30. Erkanli S, Kayaselcuk F, Kuscu E, Bagis T, Bolat F,Haberal A, et al. Expression of survivin, PTEN and p27 in normal, hyperplastic, and carcinomatous endometrium .Int J Gynecol Cancer. 2006 May, 16 (3): 1412-18.

31. Tantbirojn $\mathrm{P}$, Triratanachat $\mathrm{S}$, Trivijitsilp $\mathrm{P}$, Niruthisard S (2008). Detection of PTEN immunoreactivity in endometrial hyperplasia and adenocarcionoma. J Med Assoc Thai 2008 Aug 91:1161-1165.

32. Sarmadi S, Izadi Mood N, Sotoudeh K, Tavangar SM (2009). Altered PTEN expression; a diagnostic marker for differentiating normal, hyperplastic and neoplastic endometrium. Diagn Pathol 2009 Nov, 4:41

33. Nehad m.r. abd el-maqsoud et al Differential Expression Patterns of PTEN in Cyclic,Hyperplastic and Malignant Endometrium:Its Relation with ER, PR and Clinicopathological Parameters Journal of the Egyptian Nat. Cancer Inst., Vol. 21, No. 4, December: 323-331, 2009

34. Afaf T. Elnashar et a .Immunohistochemical expression of phosphatase and tensin homolog in endometrial hyperplasia and carcinoma Egypt J Patho 2011; 31:2-5

35. Samah Mohamed Attia et al. Immunohistochemical study of PTEN and surviving in endometrial proliferative lesions Egyptian Journal of Pathology 2011 Sep, 31:45-53.

36. Lee H, Choi HJ, Kang CS, Lee HJ, Lee WS, Park CS. Expression of miRNAs and PTEN in endometrial specimens ranging from histologically normal to hyperplasia and endometrial adenocarcinoma. Mod Pathol. 2012 Nov;25(11):150815. doi: 10.1038/modpathol.2012.111. Epub 2012 Jul 6.

37. Summayya Shawana et al Immunoexpression of Cyclin D1 and PTEN in Various Endometrial Pathologies.Journal of the College of Physicians and Surgeons Pakistan 2016, Vol. 26 (4): 277-282.

38. Orbo A, Nilsen MN, Arnes MS, Pettersen I, Larsen K. Loss of expression of MLH1, MSH2, MSH6, and PTEN related to endometrial cancer in 68 patients with endometrial hyperplasia. Int J Gynecol Pathol. 2003 Apr;22(2):141-8.

39. Cirpan T, Terek MC, Mgoyi L, Zekioglu O, IscanO, Ozsaran A. Immunohistochemical evaluation of PTEN protein in patients with endometrial intraepithelial neoplasia compared to endometrial adenocarcinoma and proliferative phase endometrium.Eur J Gynaecol Oncol 2006; 27: 389-92.

40. Kapucuoglu N, Aktepe F, Kaya H, Bircan S, Karahan N, Ciris, M. Immunohistochemical expression of PTEN in normal, hyperplastic and malignant endometrium and its correlation with hormone receptors, bcl-2, bax, and apoptotic index. Pathol Res Pract. 2007 Feb, 203:153-62.

41. Allison KH, Tenpenny E, Reed SD, Swisher EM, Garica RL. Immunohistochemical markers in endometrial hyperplasia: is there a panel with promise? A review. Appl Immunohistochem Mol Morphol. 2008 Jul;16(4):329-43. doi: 10.1097/PAI.0b013e318159b88e.

42. Kurman, R.J., Kaminski, P.F. \& Norris, H.J. The behavior of endometrial hyperplasia. A long-term study of "untreated" hyperplasia in 170 patients. Cancer, $1985 ; 56,403-12$.

\section{How to cite this article?}

Sharda B., Malik R., Jain P. Altered PTEN expression as a diagnostic marker for the earliest endometrial precancerous changes. Int J Med Res Rev 2017;5(05):515-523. doi:10.17511/ijmrr. 2017.i05.13. 